\title{
Interdependence as a Frame for Assistive Technology Research and Design
}

\author{
Cynthia L. Bennett \\ University of Washington \\ Seattle, WA \\ bennec3@uw.edu
}

\author{
Erin Brady \\ Indiana University (IUPUI) \\ Indianapolis, IN \\ brady@iupui.edu
}

\author{
Stacy M. Branham \\ University of California, Irvine \\ Irvine, CA \\ sbranham@uci.edu
}

\begin{abstract}
In this paper, we describe interdependence for assistive technology design, a frame developed to complement the traditional focus on independence in the Assistive Technology field. Interdependence emphasizes collaborative access and people with disabilities' important and often understated contribution in these efforts. We lay the foundation of this frame with literature from the academic discipline of Disability Studies and popular media contributed by contemporary disability justice activists. Then, drawing on cases from our own work, we show how the interdependence frame (1) synthesizes findings from a growing body of research in the Assistive Technology field and (2) helps us orient to additional technology design opportunities. We position interdependence as one possible orientation to, not a prescription for, research and design practice - one that opens new design possibilities and affirms our commitment to equal access for people with disabilities.
\end{abstract}

\section{CCS Concepts}

- Human-centered design and evaluation methods.

\section{Keywords}

Interdependence; assistive technology design.

\section{INTRODUCTION}

"No one is actually independent; we are all actually interdependent. The difference between the needs that many disabled people have and the needs of people who are not labeled as disabled is that non-disabled people have had their dependencies normalized. " - Ki'tay Davidson In Assistive Technology (AT) research, "independence" is often taken for granted as the goal of our contributions. For example, the gold standard of wayfinding applications for people who are blind has long been "independent navigation" $[10,18,29]$. Even when the goal is not explicitly stated, researchers and practitioners tacitly agree that "all accessible computing approaches share a common goal of improving independence, access, and quality of life for people with disabilities" [60]. But what does

Permission to make digital or hard copies of all or part of this work for personal or classroom use is granted without fee provided that copies are not made or distributed for profit or commercial advantage and that copies bear this notice and the full citation on the first page. Copyrights for components of this work owned by others than the author(s) must be honored. Abstracting with credit is permitted. To copy otherwise, or republish, to post on servers or to redistribute to lists, requires prior specific permission and/or a fee. Request permissions from Permissions@acm.org.

ASSETS '18, October 22-24, 2018, Galway, Ireland

(C) 2018 Copyright is held by the owner/author(s). Publication rights licensed to ACM.

ACM ISBN 978-1-4503-5650-3/18/10 _\$15.00

https://doi.org/10.1145/3234695.3236348
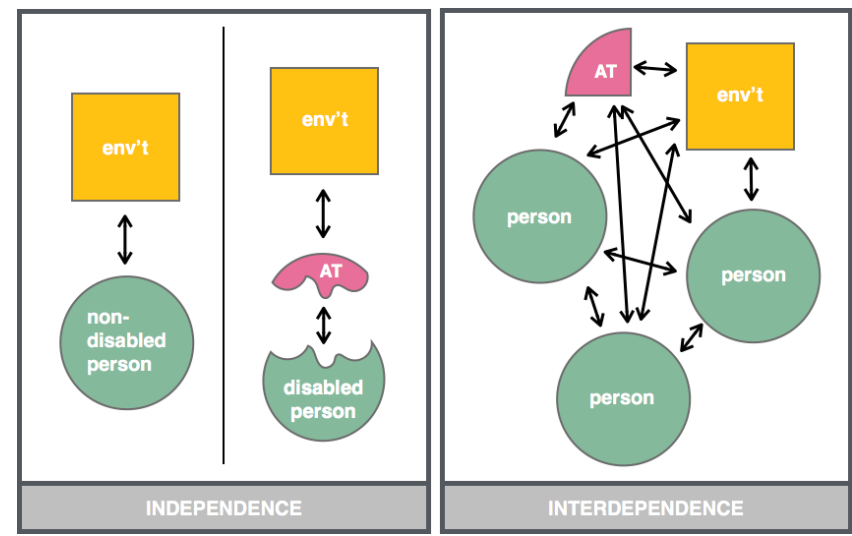

Figure 1. An independence frame (left) emphasizes an individual's relationship with the environment. Assistive technology (AT) devices are meant to bridge a perceived gap between disabled bodies and environments designed for nondisabled people. An interdependence frame (right) emphasizes the relationships between people, ATs, and environments, drawing out the roles of those with disabilities during collective work they do to create access.

"independence" really mean, especially in the context of AT? To our knowledge, there has yet to be an exploration of this kind in the AT community. The parallel development of two discursive trends within and outside the AT community suggest these are timely and consequential questions to raise.

One trend comes from outside the AT community. Disabled activists and scholars from disciplines spanning Disability Studies and Rehabilitation Medicine have begun to interrogate the meaning of independence, identify its potential negative impacts, and offer complementary framings $[12,17,21,22,25,32,35-38,40,41,58]$. For example, Science and Technology Studies scholar Aimi Hamraie argues that an independence frame "ignores the fundamental interdependence of all bodies for sustenance, community, and care" and perpetuates systemic marginalization of people with disabilities ([16], emphasis added).

A second trend comes from within the field of AT. Recent publications have begun to document the significance of social factors of assistive technology use, in ways that suggest "independence" does not always describe user behavior or goals. For example, recent studies report that device use and adoption can be affected by negative social stigmas around having a disability. Specifically, assistive devices can portray users as vulnerable or incapable, leading to awkward social interactions and minimal use even when 
the technology enables autonomy $[7,30,43,48,49,54,64]$. At the same time, AT researchers have documented people with disabilities working together with others to improve access at work $[7,8]$, during team sports [11], in face-to-face communication [13], and when using educational materials [55]. Other work described the fulfillment and joy disabled people found in helping others [27,54]. In these studies, access is not only a solution to a disability-related barrier; it is a way of being together and helping one another.

We are interested to explore why so many disability experts believe "independence" - a term used widely in our field (even by the authors of the current paper) - could be considered misrepresentative or even harmful. We believe these parallel academic explorations have synergistic qualities that form a foundation for future research and design. Specifically, we propose the adoption of the term "interdependence" to describe and synthesize recent social insights from AT researchers, using language championed by disability scholars and activists. Rather than eschewing independence, we believe interdependence can expand and explore the varieties of ways one can be "not dependent."

We begin our exploration by situating independence and interdependence within historical and political contexts. We then propose four ways that interdependence can enrich our research: (1) by allowing us to see how people and things are connected, (2) by helping us to see simultaneous forms of assistance in action, (3) by revealing often-underrepresented contributions by people with disabilities, and (4) by destabilizing traditional hierarchies that rank abilities. We present three cases from our own work in the AT field that illustrate the application of an interdependence framing. We show that many recent studies within the AT field have already been aligning with these tenets, though this paper represents the first attempt to bring them together under a single frame, and we conclude with ideas for how interdependence can be applied in accessibility research.

\section{DISABILITY, INDEPENDENCE, AND INTERDEPENDENCE}

\section{Models of Disability}

Because the meaning of "disabled" can vary widely, we begin with a brief explanation of how we use the term. We draw aspects of our definition from the evolving social model of disability. This model was first articulated by disability rights activists, and later introduced to the field of Disability Studies by Mike Oliver [42] to better represent views of people with disabilities. Oliver contrasted the social model with the individual model, the prominent conception of disability at the time. This model asserts that disability is a dysfunction of the body that should be cured, and that it is an individual's responsibility to adapt to an inaccessible world. In contrast, Oliver's social model locates disability within society, and asserts that quality of life for disabled people can be increased by removing physical and social barriers, improving attitudes, and anticipating the presence of people with disabilities in design and planning.
While the social model is a valuable tool for understanding how disability is tied to society, we agree with scholarly critiques which seek to re-emphasize the body as an important participant in disabling interactions. In this paper, we adopt Alison Kafer's political/relational model of disability, which she identifies as a "friendly departure" from the social model [21]. This model views disability as not occurring within an individual or infrastructure, but instead produced through interactions.

One important feature of Kafer's model is its recognition that the category "disabled" shifts based upon material and social context. For example, the disabling impacts of a blind person attending a primarily visual public presentation can vary depending on the overall culture around disability, the relationships they have with other attendees, their level of comfort disclosing their disability, and efforts on the part of others involved to make the presentation accessible. In other words, disability is not located within the blind person or the presentation slides, but in the blind person's relationships to other people, objects, and customs.

Some AT research reflects a political/relational model of disability. One study [49] showed AT dysfunction led both users with disabilities and those around them to relate technological error to the disabled person, decreasing confidence in that person's abilities. Here, we can see how disability is created through relations among people and assistive technologies to form assumptions about the user's capabilities. Similar to our proposed interdependence framing, we find the political/relational model of disability generative for AT research.

\section{Disability and Independence}

For people with disabilities, asserting independence has been and remains an important step toward equal rights. Suitably, the activist efforts of people with disabilities during the larger US civil rights movement of the 1970's was called the "Independent Living Movement." The Independent Living Movement declared that all people have equal worth, all people can and should make choices about their lives, and people with disabilities have the right to participate in public to the degree that nondisabled people can [2]. In the context of this movement, "independence" did not mean that a disabled person should do things autonomously - as the dictionary definition of the word implies [67] — but that supports and structures should be in place so people with disabilities can make their own choices about their futures $[21,28,46,47]$.

This definition of independence was a reaction to a long history of state- and culturally-sanctioned dependence. Historically, people with disabilities have often been isolated from society, either living at home under the care of family or living in institutions in the care of the state-where "care" often involved egregious human rights violations [20,23]. Prior to the Independent Living Movement, few government programs offered funding for in-home care services, or "attendant care," and public places included architectural 
barriers which made it difficult to access everything from supermarkets to workplaces. The movement transformed how disability was interpreted and supported outside communities of disabled people [2,14] and led to significant legal advances and protections for people with disabilities.

AT researchers seek to support independence through assistive technologies like screen readers and switches; now some mainstream smartphones and computers include builtin text-to-speech and can be easily connected to various input and output devices. Recent work recognizes people with disabilities as makers who regularly customize their assistive technologies, seeking to increase inclusion in inaccessible makerspaces $[3,34,44]$. These examples demonstrate the positive impacts of an independence frame on advancements in AT.

\section{Interdependence}

While acknowledging the importance of the Independent Living Movement, some Disability Studies scholars and activists critique its focus on independence as the main goal of accessibility [25,50]. Disability justice activist Mia Mingus argues that independence is a myth. All people constantly rely on others, even if those others are invisible to us (e.g., the people who manufacture goods we consume) [35]. Some go further to argue that emphasizing selfsufficiency can do harm to all people, disproportionately impacting people with disabilities [12]. Amid critiques, some scholars have adopted a view that interdependent relationships are necessary to achieve access.

While the term interdependence has not been used in AT literature, the concept has long been discussed in Disability Studies and disability justice circles. For example, in the very first issue of the Disability Studies Reader, published in 1997, Feminist Disability Studies scholar Susan Wendell argued that a culture based in "interdependence" as opposed to "self-reliance" would improve quality of life for disabled people [57].

Even before the term was adopted, interdependence was being practiced by disability activists. "Independence" was central to the Independent Living Movement, yet modes of disability activism were deeply interdependence-focused. For example, the first Center for Independent Living (CIL) consisted of a collective of UC Berkeley volunteers led by students with disabilities. They taught each other strategies for advocating access and living on their own, removed structural barriers around campus, ran a wheelchair repair shop, and built a network of trusted attendant care providers that disabled students could hire [47]. British disabled activists adopted the Western CIL model, but prefaced the names of their "living centers" with "integrated" or "inclusive", instead of "independent," to acknowledge these social aspects of access [2]. In other words, to achieve the goal of independent living, activists relied significantly on interdependent relationships with one another.

Modern activist groups, like The Disability Justice Collective in the San Francisco Bay Area, use the term "interdependence" to represent ways they need one another. Led predominantly by LGBTQ people of color, their experiences with multiple oppression necessitate reliance on community support and collectively-done access [25,3537,50]. Part of the collective's work is to help disabled community members find trustworthy access partners. For example, their disability justice primer suggests sharing routes home to stay safe, dividing access-related tasks according to group members' strengths, and being flexible to dynamic access needs such as offering remote attendance [50]. This type of close relationship development in the process of providing interdependent access has been called access intimacy by disabled activist Mia Mingus [37].

\section{Independence or Interdependence?}

Given the universally positive presentation of interdependence above, it may seem that we always advocate interdependence over independence. We do not for two reasons. First, independence and interdependence are not dichotomous or mutually exclusive. Rehabilitation medicine scholars [58] recommended that vocational rehabilitation programs for people with disabilities teach interdependence alongside independence. They argue that teaching independence empowers people with disabilities to take control over their access needs. But, instilling interdependence establishes them as contributors to-not just recipients of - community support and assistance.

Second, interdependence is not necessarily better than independence, and the following examples elucidate these complexities. Disability Studies scholar Christine Kelly [22] described the possibilities and pitfalls of interdependence in her "frien-tendant" relationship. She is sometimes paid to provide care for a man, Killian, who is also a close friend. Their companionship has honed an attunement among them, elevating her job responsibilities from completing instrumental tasks to an interdependent mingling of providing care and being together. Their partnership brings out Killian as not a passive recipient of care, but equal and active in cultivating friendship. Yet, gendered histories of power make Kelly feel vulnerable and out of place when she takes Killian into men's restrooms.

Another example shows how reliance on AT can sometimes lead to unfortunate outcomes. Science and Technology Studies scholars Moser and Law [41] described how a wheelchair user's travel plans went awry when their train arrived with a broken wheelchair lift. In this case, the user's interdependent relations with unreliable AT and social institutions that tolerate trains without functional wheelchair lifts prevented their self-determination of mobility.

These samples show that we can make sense of both positive and negative experiences through an interdependence lens. In other words, interdependence does not ensure access. Yet, as we will argue in depth below, the framing helps illuminate new possibilities for AT design. Focusing solely on independence - on whether an individual achieves a task autonomously - leaves out important factors that can help us to for example, anticipate the awkward aspects of social 
interactions and the roles of policy, labor, and materials that shape infrastructural-level decisions [17]. In the following section, we define interdependence again, this time in terms orientated to the AT community.

\section{DEFINING INTERDEPENDENCE FOR THE ASSISTIVE TECHNOLOGY FIELD}

We now present four tenets to summarize what an interdependence frame can reveal in our AT research. We have listed them here and explicate each below. An interdependence frame (1) focuses on relations, (2) helps us make sense of multiple forms of assistance happening simultaneously, (3) draws out the often-underwritten contributions of people with disabilities, and (4) can help disassemble hierarchies that prefer ability.

\section{Interdependence Allows Us to See Relations}

The definition of the term interdependence implies a relationship between people and things and focuses our attention on what goes on within their interactions. We do not intend for the word "relations" to indicate a long-term history of interactions - rather, "relations" refers to a coming together of people and things in a particular moment in time. In articulating the political/relational model, Alison Kafer describes disability not as a static entity but as "events, actions, and encounters" [21] produced through interactions. Similarly, interdependence centers relations between those things that make and define disability and access. With an interdependence framing, we can characterize the accessibility of a situation with respect to a broader range of influential factors that lead to them.

In AT research, studying relations can help nuance the individual experiences often reported in our findings. Notably, researchers like Shinohara and Wobbrock $[43,48,49]$ have studied how social interactions that involve apparent markers of disability, like assistive technologies, impact both people with and without disabilities.

The important relations that can be identified through an interdependence framing do not just include relations between people and assistive technologies, but also those between people in situations where assistive technologies are not used. For example, AT researchers [59] conducted observations of blind and sighted partners navigating new environments together. This work identified ways in which much navigation research seeks to facilitate "sighted" styles of navigation for blind travelers, and why this may be challenging or dangerous because of sighted people's misconceptions of blind navigation. Important for understanding navigation mishaps is examining the way sighted partners were trying to relate to their blind partner. When challenges arose, they mapped their experience onto their partners' and subsequently provided instructions they would have appreciated should they have been the ones disoriented. Studying how everything and everyone involved in assistance is relating with one another can emerge opportunities for supporting smoother interactions.

\section{Interdependence can Reveal Simultaneous Relations and Assistance}

Identifying relevant relations will provide insight into interactions, but interdependence also makes available the possibility that there might be simultaneous relations impacting a particular moment. Disabled activists including Nomy Lamm [25] who write about collective access describe it as an ongoing process where everyone supports one another. This means that some people provide access support while receiving it, and multiple types of access support are provided during one event. An interdependence framing can involve breaking down assistance acts to learn how each participant is providing and receiving assistance.

By attending to simultaneous relations, we can also view how else people are connecting during assistive interactions. Again, Kelly's autoethnography of her [22] "frien-tendant" relationship with Killian is illustrative. "Frien-tendant" thoughtfully described their relationship as more than the mechanical acts of assistance that could be performed by any attendant interchangeably. She explicitly acknowledged the ways in which Killian simultaneously supports her through their relationship, acting with care to identify what she is capable of providing and how it will impact their friendship.

In AT research, work like Branham and Kane's exploration of mixed-ability co-habitants [8] demonstrates assistance as more complex than a solution to a problem. Assistance and relationship maintenance were intertwined-while nondisabled partners were happy to help their partners with disabilities, both parties sometimes found acts of assisting interfered with other important factors in their relationship. Relationships between people can pre-date requests for access help, or become more complex through assistive interactions themselves. Studying simultaneous relations in the context of AT research can reveal opportunities to support relations that intertwine with assistive interactions.

\section{Interdependence can Reveal the Work Done by People with Disabilities}

Since interdependence considers everyone and everything in an interaction to be mutually reliant, this framing can foreground some of the historically-ignored (but extremely important) work done by people with disabilities. Traditionally, people with disabilities are portrayed as recipients of, not agents in, securing their assistance [20]. But the Independent Living Movement thrived on activists with disabilities helping one another, and their demands influenced law and policy which impacted many people outside the movement itself. Interdependence can reveal the work that goes into access holistically, including the important, overlooked work done by people with disabilities.

An interdependence framing would not take the presence of accommodations in a building - such as wheelchair ramps at the entrances or AT software installed on computers - as evidence of "access." Instead, these tangible accommodations should be studied in concert with sociotechnical considerations. Access in this setting also 
includes how people with disabilities operate with technologies, communicate their needs, and manage their AT-mediated interactions in hopes of presenting themselves professionally. An interdependence framing can help us more adequately represent everyone's needs and contributions and encourages us to focus more attention on the practices that people with disabilities engage in to cocreate accessibility with their peers.

This work done by people with disabilities to secure access has previously been described in AT and feminist-oriented HCI research as "invisible" [7,52]. Branham and Kane [7] documented the ways blind employees do their jobs in mixed ability workplaces. Sighted employees pervasively misunderstood access needs, and office spaces and cultures still proved inaccessible. The blind participants' accounts demonstrated the lengths that blind employees undertook, largely unknown to sighted employees, to maintain awareness of inaccessible aspects of work. Blind employees also had to invest time and energy in educating colleagues on how best to assist them. Rather than attributing access and accommodations to the presence of support resources in a space, interdependence ensures that we are also giving significance to the work people with disabilities do to communicate and co-create accessibility.

\section{Interdependence can Challenge Ability-Based Hierarchies}

Finally, since interdependence considers everyone mutually reliant, it asserts that people with and without disabilities are equal. Traditionally, society has viewed people with disabilities as being worth less than their nondisabled counterparts [15], so their actions and contributions are often ranked lower in a hierarchy of importance. This ordering arises from the prominent and problematic narrative that assistance provides mechanisms through which people with disabilities become more able, more whole, more "normal"-essentially, more like non-disabled people [38].

Science and Technology Studies scholar, Ingunn Moser, interviewed people with disabilities about their ATs showing how the technologies themselves, along with factors such as government resources, high cost, and disability-related stigmas, undermine people with disabilities. Their efforts to navigate complex insurance and government-funded programs to justify their technology needs, and the skill to operate specialized interfaces went unrecognized in favor of focusing on the liberation such programs and devices provided $[39,41]$. Interdependence seeks to break out of this circular problem by explicitly valuing people, including those with disabilities, from the outset for who they are and irrespective of their abilities or assistive technologies they use. Instead, they are assumed integral to interdependent interactions for bringing valued perspective and skill regardless of whether they are assisting, being assisted, or doing both. With this in mind, an interdependence framing can help us to contribute research that pushes back on these histories with not only accessible designs but also designs which give attention to and challenge ability-based hierarchies.

Prior AT research has worked to challenge these implicit hierarchies and biases across different domains. The designers of Incloodle [51] observed that neurotypical children often excluded neurodiverse children from play activities, and designed a cooperative photography application to enforce equal participation among participants to counter this hierarchy. Other work explored the potential of people with disabilities as employees [65] or volunteers [24,27], recognizing their unique strengths rather than immediately classifying people with disabilities as "less than." These papers begin the hard work of challenging hierarchies that devalue disabilities in favor of more abilities.

\section{APPLYING THE INTERDEPENDENCE FRAME: THREE CASES}

Using these tenets, we present three cases drawn from our research on the accessibility practices of people with various disabilities. We chose these cases to represent a diversity of contexts, disabilities, and scopes. Collectively, they exemplify the flexible application of an interdependence frame to interpret how accessibility is made by people, including those with disabilities, in everyday situations.

\section{Case 1: Organic Interdependence on Aisle 3}

The first case draws from field observations of two people shopping together. The couple, William and Emily, are both visually impaired. Emily has no sight and she navigates with her guide dog, Jasmine. William has a full field of very little sight and he navigates with a white cane. This case was one of several observations of finite interactions of assistance, hence data collected are in the form of a conversation transcript. Below is an excerpt from the transcript of William, Emily, and Jasmine, exiting a store after purchasing items. We then share how an interdependence framing sensitizes us to the four above tenets.

Emily: Do you want me to find the door?

William: Yeah go on then yeah.

Emily: Where have we gone now? [Emily and Jasmine begin to walk right down an aisle]

William: I think we've... Oh, we've headed up to the perfumes.

Emily: 'scuse me. [Walks around someone].

Emily: [speaking to Jasmine] Good girl. [to William] Where are we going?

William: Are we lost?

Emily: Yeah, ... errrr... [Turns left] (to Jasmine) Straight on. Find the door. Good girl, find the door. Find the door, good girl [Emily and Jasmine stop about 4 meters in front of door]. Oh, it doesn't open.

William: It's not an automatic door. [Walks around Emily and Jasmine and opens swinging door] 
Emily: Oh, isn't it? [Walks through as William holds door open]. Oh, hehahehaheha. We got stumped by a door.

\section{Relations}

It is clear from this vignette that William, Emily, and Jasmine are in relation with one another and the store to find the exit. Through conversation, they asked each other for help, share access information, and check on one another's understanding of their progress. Additionally, they related to things around them and their memories to find the door.

\section{Simultaneous Relations and Assistance}

This scenario shows how relating to one another and the environment made finding the door possible. Yet it is impossible to understand the process without considering that William, Emily, and Jasmine were simultaneously relating and providing access. Each was using the resources available to them to make sense of the environment for everyone. In this way, William, Emily, and Jasmine each scaffolded assistance. Specifically, Emily established that she would locate the door-but she was assisted by William who grounded their location after smelling and visually recognizing perfume counters, from Jasmine who recalled the direction of the door after Emily's command to find it, and again from William who opened the door. Each took information from the other to generate new information, and trying to understand anyone's relationship in isolation would omit important details of how they left the store.

Additionally, to understand the assistance, we have to learn about the other relationships. In this case, we see evidence of access intimacy where Emily and William and Emily and Jasmine work well together. William and Emily did not have to tell the other when they detected that their navigation strengths might be useful, or when the other might know what to do next. Instead, they switched guiding one another seamlessly. Additionally, Emily has undergone extensive training and years working with her guide dog, and as partners, the two have developed attunement such that Emily could give Jasmine commands mid conversation with William. Considering the multiple relationships helps us to understand the assistance as situated and personal to them.

\section{Visible Work and Challenging Hierarchies}

One unique aspect of this case when considered alongside research on guidance is that both William and Emily are visually impaired. This foregrounds the important work each did to assist in finding the exit. Without full access to the numerous visual cues that may have orientated them, they instead leveraged resources they could make sense of. Warren could smell perfumes and slightly make out display counters, and their navigation aids helped them to avoid obstacles. An interdependence framing also classifies the nondominant strategies they used as legitimate information sources to draw from in providing assistance. Recognizing this helps us to not compare their strategies to those that sighted people might use, but to think about how technology may support strategies that already work for them.
This conversation provides a momentary snapshot from an entire day of shopping. But analyzing how William and Emily communicated through an interdependence lens revealed rich insights from even a short transcript. We witnessed their multiple relationships with one another and their environment working in concert toward helping them to find the door, and how they leveraged orientation and navigation strategies unsupported by the store's cues assuming customers would have full vision. Taking any aspect of their interaction on its own would leave out important factors that contributed to William and Emily's accomplishment. Instead, it was their relations with one another and their environment along with undertaking strategies available that revealed informative nuances of a seemingly unremarkable act of exiting a store. In that moment, William and Emily did not want tools to emulate vision, and they did not consider assistance separate from just being together. Rather, our research could pinpoint specific ways to support their process.

\section{Case 2: Working Out Interdependent Strengths}

The second case comes from a professional workplace in the public sector. The scope of study was three members of a team comprising roughly ten colleagues, who work closely with one another in nearby cubicles. The researchers conducted interviews and observations to assess the accessibility practices in the office.

Disability and access were central to the identity of the team in two ways. First, the team's mission was to oversee disbursement of grant funding to address workplace accommodations and accessibility initiatives for state and local governments. Second, every member of the team identified as having a disability. The three members who worked most closely with one another represented a wide spectrum of ability: one was blind (Abby) and accompanied by a guide dog (Ali), another was hard of hearing (Bruce) and used hearing aids, and another was a wheelchair user (Crissy). In the context of the organization, Abby was most senior. Bruce reported to Abby as her reader for half of his daily duties. Crissy had more seniority and responsibility than Bruce, but was a slightly more junior mentee of Abby. This team was one small part of a much larger organization in which people with disabilities were a minority.

Below, we explore how these team members encountered and collaboratively approached accessibility challenges in the office. We draw out the tenets of interdependence from observations of work practices, and through participants' own accounts of their relationships with one another.

\section{(Simultaneous) Relations}

Work in the office was often collaboratively shared according to the strengths of each team member and negotiated to bring activities in alignment with interests of the collective. For example, Abby and Crissy often practiced what Crissy called "cross-ability cooperation." 
Crissy: "I will come [to the vending machine] when she wants to, and I'll go 'well, what do you feel like?' and she'll tell me what she feels like and I'm like, 'on this row, they've got-', you know and I'll describe the different products, and then I'll go 'which one do you want?' and I'll push the button for whichever one it is and Abby will bend over to retrieve the snack."

Abby: "We developed a collaborative cafeteria plan. We'd go downstairs and scare the hell out of people because I'd push her wheelchair or get food for her and carry stuff back for her. 'Crissy, do I [Abby] have schmutz on my pants?' That takes a lot of time and friendship to get to that arrangement."

Crissy and Abby developed a relationship in which they are familiar with each other's abilities and find ways to work together that play to their strengths. Crissy described visual information while Abby pushed Crissy's wheelchair and grasped out-of-reach items. But their interdependence did not come immediately or outside other relationships. It took Abby a long time to feel comfortable enough with Crissy to inquire about her appearance after eating. Though the pair embraced "scaring the hell out of people," Abby acknowledged that their interdependence with visible disabilities is an uncommon sight, inviting attention from passersby who question their suitability to work together.

Abby and Crissy were not just assisting one another; they operated in more complex relations with other colleagues who offered different complementary strengths:

Abby: "I often give Bruce a spreadsheet and say, 'this is what I want you to track in it.' Crissy tries to do it herself [but she has trouble tracking]. It took me a while to get there, if I was younger it would have felt like a cop out [to ask Bruce for help]."

Abby delegated work that both she and Crissy could technically do, but not as efficiently or accurately as Bruce. Abby was aware that when she was less comfortable with her own strengths and weaknesses, she would have felt it a "cop out" to delegate. But, she felt a security in both her career and her disability that allowed her to lean into interdependent relationships to achieve access.

Even when Abby worked with a nondisabled colleague in another unit of the company, an interdependence framing helps us see the contributions made by both parties:

Abby: "I've worked with [sighted worker] for years. We've become good friends, because I started here 6 years ago, and I couldn't read the grant awards, the budgets. I called her up and asked her. Just recently, her whole team went to an accessibility training in order to try to improve their PDF documents."

Interviewer: "So, it helps to have friends over there."

Abby: "It does. Well, it helps to become real. You know, there's an image of accessibility, there's an image of 'this is the right thing to do.' But, when you have a person who needs access and that person is real to you, then you're going to try hard."

Here, we found that Abby worked to develop relationships with colleagues who might be seen as gatekeepers to access. Through six years of ongoing engagement, Abby was able to demonstrate to remote colleagues that she was a "real" person who could be included in collaborations if only they would make small changes to workflows like creating accessible PDFs. The outcome of her ongoing labor resulted in interdependency with nondisabled colleagues who began producing documents accessibly from the start.

\section{Visible Work}

In the examples above, making visible the work done by people with disabilities is integral for researchers and the participants. Visibility not only helps researchers understand co-creation of access more holistically, but it led Abby's colleagues to humanize access from abstract guidelines to an essential component for blind employees to do their job. This increased understanding then motivated the colleagues to integrate accessible pdf production into their workflow. Finally, it is important to recognize the interdependency these colleagues have with office culture that is friendly to communication, collaboration, professional development, and working relationships that do not rank disability lower. As elaborated below, this culture is what makes articulating access work possible.

\section{Challenging Hierarchies}

The three coworkers reported to supervisors in a traditional managerial hierarchy. Abby was forthcoming that her seniority increased her comfort to ask for and accept help from colleagues. An interdependence reading of their collaboration, however, illuminates how their situation both perpetuates and disrupts traditional professional and abilitybased hierarchies. For example, despite her seniority, it took Abby six years of advocacy to convince sighted colleagues to produce accessible PDFs. Her persistent advocacy and ongoing friendship with an employee on the team led her colleagues to get training to produce accessible documents.

Participants made clear that accessibility was achieved "together," "with" each other, and without one partner controlling the other. Abby expressed this in many ways:

"I couldn't attach two, three, or more attachments because the buttons became hidden to JAWS [screen reader]... [Bruce] told me where I was without trying to take over."

"Instead of worrying about people saying, 'he's doing it for her.' He's not. He's doing it with me."

We find here that Abby did not rank herself with Bruce according to their abilities; in fact, she considered such a metric irrelevant when they could do something together. These examples show how interdependence allows people with heterogeneous abilities to visibly pool their strengths to achieve shared access. Moreover, like the disability justice activists, interdependence was not an abstract frame for researchers to apply to observations; participants lived collaborative accessibility and described it in such terms. 


\section{Case 3: Interdependent Activism at a Distance}

The 2017 Women's March on Washington was a protest of Donald Trump's election as president despite a history of anti-women behavior and politics. Planning for the protest spread wildly via social media, and Women's Marches were held simultaneously in over four hundred cities in the United States on January $21^{\text {st }}, 2017$. The event promoted resistance among women who might be impacted by policies limiting reproductive rights, LGBTQ+ rights, and healthcare reform.

However, a "march" demands that participants be able to execute a certain set of physical abilities (including standing and moving throughout the streets) and involves many sensory stimuli that might trigger symptoms. As a result, this "Women's March" by nature was not inclusive of women with mobility impairments, weaker immune systems, and certain mental health conditions.

Accessibility problems were compounded by the abilityspecific rhetoric used to describe the march on social media. Organizers and attendees highlighted the importance of physical presence at the march and the need to "stand together in solidarity" [66]. In reflection, many Women's March attendees again emphasized the importance of their attendance in person, commenting that "I just felt so empowered being there" or "I felt the need to show up with my body and my voice." ([33], emphasis added).

Frustrated by the inaccessibility of "marching" and hoping to ensure representation of women with disabilities in the movement, a group of mostly-female advocacy writers (who were already connected online around their shared health conditions) conceptualized and ran a "Disability March." The Disability March was a blog and social media platform where disabled women and their allies could asynchronously share stories of resistance against misogynist and ableist structures in society. Building on prior work [26], we present the Disability March as an example of collective action that embodies the major concepts of interdependence.

\section{(Simultaneous) Relations}

The Disability March demonstrates a great deal of the relational work of interdependence amongst the march's participants, observers, and organizers. Participants attended the Disability March to join in the communal expression of outrage that participation in the Women's March signified, and to support the marchers attending the event in person. Often, they invited other people with disabilities from their own networks to join the Disability March, broadening the reach of their own stories and building the collective. By joining the Disability March, these individuals both contributed to and were shaped by the larger movement.

The Disability March was created to reach a wide audience of observers, consisting of both people with and without disabilities. This mixed audience again fosters the mutual reliance that is core to interdependence. People with disabilities contributed their stories, sharing their unique lived experience with people without disabilities who might not have access to their expertise otherwise.
Additionally, people we spoke with highlighted the ways in which they simultaneously participated in non-digital activism. Some described how they leveraged existing access intimacies to attend in-person events with their families or friends. While their companions often had to take the role of "caregiver" in these travels, their attendance was mutually reliant, building a larger coalition together. Others found opportunities to generate new access intimacies with strangers around their shared cause. For example, one participant described a new form of collective activism:

'Last night I found a group called 'Walk with Us' where they match a disabled person to go [to a rally or protest];

instead someone else can go in your place. They take a picture of you and you sit and get connected with them...."

This project fostered connections between members of the movement, strengthening its coherency and broadening its presence. Many organizers began as participants, but were drawn into more formal roles in which they could support the organization through the skills they brought with them.

\section{Visible Work}

All of the work of the Disability March was conceived, organized, and executed by people with disabilities, and the two core organizers we spoke with identified as female and queer, respectively. These organizers operated as agents of accessibility, rather than recipients of it, developing a satellite movement to fit their own needs and abilities. However, this process required a large amount of invisible work and coordination among organizers, which may not have been recognized by most Women's March participants.

The Disability March was initiated by members of an existing collective of writers with chronic illness. This structure allowed them to leverage their existing expertise and practices for ensuring accessibility (e.g., including text descriptions of all images posted on their website) to the design of the platform. Rather than striving for a march experience that directly replicated the physical march, these organizers identified the critical roles of asynchrony and multimedia in communicating around accessibility and embedded these values into their platform. This march was likely more successful because of the insights contributed by disabled organizers and helped the original Women's March better recognize the limitations of in-person marching.

\section{Challenging Hierarchies}

The development of the Disability March directly contradicts some of our traditional, hierarchical understandings of "assistance" and "care." First, the Disability March itself draws on the early disability rights protests - a group of activists self-organizing to ensure that their access needs were met appropriately. Examining an event with an interdependence lens gives us the awareness that people with disabilities can be organizers and initiators of movements, rather than passive participants or beneficiaries of others' activism.

The public nature of the Disability March similarly served to flatten traditional ability-based hierarchies. The Disability 
March was a tangible reminder from disabled women that their voices and their representation mattered just as much as the able-bodied women who could attend events in person. The organizers and participants recognized the importance of spreading a disabled perspective at a broader level, and one participant drew attention to the number of people with disabilities to indicate how prevalent those perspectives have become:

"That's why I was so interested to participate - because people with disabilities have so much to say, and now it is national. The Disability March has introduced [our participation] to a new level... the repercussions of the outcome of election has so much put us in the front now...."

\section{DISCUSSION}

This paper is the first attempt we know of in the Assistive Technology community to articulate the origins and meanings of the term independence as it pertains to people with disabilities. Our inquiry demonstrates the important role independence has played in orienting toward disability, specifically for advancing disabled peoples' claim to basic human rights. Our inquiry also surfaced contemporary scholarship and activism that offers a complementary frame, interdependence. Interdependence emphasizes how myriad people and devices come together to build access, with special attention to acknowledging the work of people with disabilities. We propose that an interdependence frame can synthesize recent scholarly contributions from within the AT field, which increasingly emphasize social dimensions of accessibility (e.g., $[48,49,54,59,64])$.

Applying our interdependence lens, we learn how access occurs through interactions that are not just about getting something done. Rather, access is entangled with being and doing together when shopping, working, or even protesting. Below, we share how the interdependence frame raises new opportunities for three vibrant areas of AT research.

\subsection{Crowd Work and Interdependence}

We identify two threads of ongoing research related to crowd work and accessibility that may be usefully expanded through an interdependence framing. First, AT researchers $[4,5,9,24,27,53,56,61,62,65]$ have explored crowd work as a means for people with disabilities to both receive assistance and find employment. Research regarding on-demand assistance focuses on systems, like VizWiz and RegionSpeak $[4,6,62]$, that connect a disabled person in need of assistance with a remote crowd worker. Costs associated with this model, along with exciting innovations in AI, have nudged future directions for this work toward minimizing human assistance [31,63]. As such, crowd work solutions can be seen as an early means of amassing ground truth data to train machine learning models for fully-automated tools.

A second thread of research addresses the viability of crowd work as a source of income and volunteerism for older adults $[9,24]$ and people with disabilities $[27,53,56,65]$. Findings show that members of this population are interested and capable of doing the work. Benefits of crowd work, including flexible hours and working from home, meet the needs of some workers with disabilities. However, pervasive inaccessibility of crowd work platforms significantly impedes full participation of people with disabilities.

An interdependence framing allows us to ask questions about these threads and offer new directions for assistive crowd work research in three ways.

First, whereas crowd work systems tend to consider tasks mutually exclusive, an interdependence frame might recommend crowd work systems that allow requesters and workers to build rapport and complete multiple tasks together. When requesters and task completers do not know one another, crowd work platforms might provide questions to help the requester more adequately contextualize their access need. And, because interdependence sees people with disabilities as agents in creating access, the system might recruit people with disabilities to assist other requesters with or without disabilities. Such considerations would position people with disabilities as eligible to provide and receive assistance, explicitly denoting their contributions while challenging ability-based hierarchies.

Second, current crowd work systems that provide remote visual assistance classify blind task submitters differently from (sighted) workers completing tasks. Interdependence highlights work done by people with disabilities, raising questions about who is credited for what. At first glance, the person assigned a task seems to be doing work; their title as a crowd "worker" makes this explicit. System names like "BeMyEyes" [68] minimize the role of blind individuals by framing them as passive recipients of assistance. Yet, this overlooks the significant work performed by the blind requesters to send a photo or video, work that requires them to negotiate highly visual access barriers [19]. These systems could, for example, allow blind requesters to supplement photograph submissions with contextual information that may help someone answer in absence of a perfectly framed or clear image. At the same time, though task completers are called "workers," after the completed task is returned to the requester, their contribution disappears. Interdependence could guide conversations on limitations of systems that enforce each task to ultimately have one owner and speculate new forms of assistance that honor everyone's contributions.

Finally, advancements in object recognition present OCR systems as replacements for human assistance. Yet, independence and interdependence can coexist as dual goals for accessibility. We can imagine hybrid solutions in which future systems that automate tasks still integrate human assistance. For example, Aira [31], a visual interpreting subscription-based service, released smart glasses with OCR capabilities. In this way, a subscriber can leverage automated and human assistance through the same device.

\subsection{Ability-Based Design and Interdependence}

An influential approach for designing assistive technologies has been Wobbrock et al.'s Ability-Based Design [60]. 
Ability-Based Design contends that technologies should be responsive to each user's unique interaction capabilities, and that AT researchers should design systems that are appropriate for multiple users and contexts. Because AbilityBased Design focuses on users' independent interactions with systems, other people and devices who enact assistance are grouped in the broad category of "context." With an interdependence lens, we can expand Ability-Based design's call for adaptive user interfaces to include considerations of the relationships people have with contexts-their disabilities, their devices, and other people.

Our cases show that relationships between these three elements of context were significant to interactions and accessibility outcomes. William and Emily's access needs changed as they traversed the store (Case 1), but their experiences relating with one another kept the navigation from presenting significant barriers. One way employees with different disabilities "hacked" accessibility was to swap tasks according to their strengths (Case 2), And participation in the Disability March changed the way some participants with disabilities viewed their own identity (Case 3 ). While some view online activities as slactivism, relating with a community of disabled remote marchers legitimized their contributions, raising their confidence that disability could represent an important perspective in political organizing.

In response, AT's could adapt according to these relations, such as when in the presence of a "frien-tendant" or in a social situation where the user does not want their disability to be apparent. Fiannaca et al.'s [13] AAC device does just that by allowing users to specify what granularity of drafted text conversation their partners can view based on their comfort level with them. Ability-Based designs might provide a feature for users to share how they are interacting with the system and when they encounter barriers; data could be used to shift misconceptions about the work people with disabilities are (not) doing [49]. In this way, the values of independence that are bolstered by Ability-Based designs can be considered alongside interdependency.

\subsection{Navigation and Interdependence}

Accessible navigation has long been a focus of AT research $[10,18,29]$. Navigation aids, whether or not developed with people with disabilities in mind, tend to focus on directing a user to a destination. While some allow users to select stops along the way, the task of navigating is largely presented as one in which a user follows instructions from a technology, with destination arrival as the metric for success.

An interdependence framing guides us toward questions focusing on the journey-specifically about what relations work together to facilitate navigation by people with disabilities. Abdolrahmani et al. [1] studied how context and error type intersect with disability to impact assistive navigation device adoption. We see relationships at work to construct different scenarios with contingent needs and preferences. For example, they found that a device guiding a blind user into the wrong store was not consequential to them, but entering the preferred bathroom for their gender identity was of paramount importance. Unlike predominant themes in AT research which attempt to minimize errors altogether, they contend that navigation systems will not be error free, so they instead direct research to support contextappropriate error and safety management processes for users with disabilities. We expand this research direction to suggest that navigation systems could combine multiple types of assistance throughout the journey to support navigation management. This could include navigation systems that allow a user to triangulate information from multiple sources. Additionally, users with disabilities could enroll access partners $[45,61]$ to suggest alternative routes or to explain segments undiscernible by technology.

Interdependence could also inform navigation by people with mixed abilities [7,8,59]. Past research [59] found crossability navigation assistance was not always joyfully done; sighted people misunderstood how to best assist blind walking partners. In the context of the home, some participants were cognizant that asking for assistance strained sighted partners leaving those with visual impairments unsure how to contribute to the relationship [8]. An interdependence framing could support collaborative assistance and well-being by pointing out opportunities for peer support. Systems might assign different access partners with complementary abilities and allocate time to check in on each other's concerns and energy levels.

We have demonstrated how an interdependence frame can widen existing areas of AT research. While interdependence can be critical of the limitations of previous independencefocused research, it is also generative, encapsulating a method for transferring the relational assistance we have observed into actionable directions for AT research.

\section{CONCLUSION}

We introduced interdependence as a complementary frame for AT researchers. Interdependence (1) considers access to be relational and (2) simultaneous, (3) highlights the contributions of people with disabilities, and (4) challenges traditional hierarchies of ability. We demonstrated the frame's benefits by applying it to three cases from our research and three emergent areas of AT research: crowd work, Ability-Based Design, and navigation. We present interdependence as a frame not to guide designing for people with disabilities from a distance, but by them, as its introduction into the ASSETS community is indebted to their lived experience, activism, and scholarship.

\section{ACKNOWLEDGMENTS}

We thank the participants from our three case studies for inspiring this paper, and Daniela Rosner and Alex Taylor for reviewing drafts. This work was supported by NSF grants \#DGE-1256082 and IIS-1353312.

\section{REFERENCES}

1. Ali Abdolrahmani, William Easley, Michele Williams, Stacy Branham, and Amy Hurst. 2017. Embracing Errors: Examining How Context of Use Impacts Blind Individuals' Acceptance of Navigation Aid Errors. In 
Proceedings of the 2017 CHI Conference on Human Factors in Computing Systems, 4158-4169.

2. Colin Barnes. 2003. Independent living, politics and implications.

3. Cynthia L. Bennett, Keting Cen, Katherine M. Steele, and Daniela K. Rosner. 2016. An intimate laboratory?: Prostheses as a tool for experimenting with identity and normalcy. In Proceedings of the 2016 CHI Conference on Human Factors in Computing Systems, 1745-1756.

4. Jeffrey P Bigham, Chandrika Jayant, Hanjie Ji, Greg Little, Andrew Miller, Robert C Miller, Robin Miller, Aubrey Tatarowicz, Brandyn White, Samual White, and others. 2010. VizWiz: nearly real-time answers to visual questions. In Proceedings of the 23nd annual ACM symposium on User interface software and technology, 333-342.

5. Erin Brady, Meredith Ringel Morris, and Jeffrey $\mathrm{P}$ Bigham. 2015. Gauging receptiveness to social microvolunteering. In Proceedings of the 33rd Annual ACM Conference on Human Factors in Computing Systems, 1055-1064.

6. Erin Brady, Meredith Ringel Morris, Yu Zhong, Samuel White, and Jeffrey P Bigham. 2013. Visual challenges in the everyday lives of blind people. In Proceedings of the SIGCHI Conference on Human Factors in Computing Systems, 2117-2126.

7. Stacy M. Branham and Shaun K. Kane. 2015. The invisible work of accessibility: how blind employees manage accessibility in mixed-ability workplaces. In Proceedings of the 17th International ACM SIGACCESS Conference on Computers \& Accessibility, 163-171.

8. Stacy M. Branham and Shaun K. Kane. 2015. Collaborative accessibility: how blind and sighted companions co-create accessible home spaces. In Proceedings of the 33rd Annual ACM Conference on Human Factors in Computing Systems, 2373-2382.

9. Robin Brewer, Meredith Ringel Morris, and Anne Marie Piper. 2016. Why would anybody do this?: Understanding older adults' motivations and challenges in crowd work. In Proceedings of the 2016 CHI Conference on Human Factors in Computing Systems, 2246-2257.

10. Megan Campbell, Cynthia Bennett, Caitlin Bonnar, and Alan Borning. 2014. Where's my bus stop?: supporting independence of blind transit riders with StopInfo. In Proceedings of the 16th international ACM SIGACCESS conference on Computers \& accessibility, $11-18$.

11. Patrick Carrington, Denzel Ketter, and Amy Hurst. 2017. Understanding Fatigue and Stamina Management Opportunities and Challenges in Wheelchair Basketball. In Proceedings of the 19th International ACM SIGACCESS Conference on Computers and Accessibility, 130-139.

12. Dom Chatterjee. 2018. Independence is an Ableist Myth: Unlocking the Power of Community in Healing.
The Body is Not An Apology. Retrieved February 22, 2018 from https://thebodyisnotanapology.com/magazine/indepen dence-is-an-ableist-myth-unlocking-the-power-ofcommunity-in-healing/

13. Alexander Fiannaca, Ann Paradiso, Mira Shah, and Meredith Ringel Morris. 2017. AACrobat: Using mobile devices to lower communication barriers and provide autonomy with gaze-based AAC. In Proceedings of the 2017 ACM Conference on Computer Supported Cooperative Work and Social Computing, 683-695.

14. M. P. A. Gerben Dejong. 1979. Independent living: From social movement to analytic paradigm. Arch Phys Med Rehabil 60.

15. Erving Goffman. 2009. Stigma: Notes on the management of spoiled identity. Simon and Schuster.

16. Aimi Hamraie. 2013. Designing collective access: A feminist disability theory of universal design. Disability Studies Quarterly 33, 4.

17. Aimi Hamraie. 2017. Building Access: Universal Design and the Politics of Disability. University of Minnesota Press.

18. Andreas Hub, Joachim Diepstraten, and Thomas Ertl. 2004. Design and development of an indoor navigation and object identification system for the blind. In $A C M$ Sigaccess Accessibility and Computing, 147-152.

19. Chandrika Jayant, Hanjie Ji, Samuel White, and Jeffrey P Bigham. 2011. Supporting blind photography. In The proceedings of the 13th international ACM SIGACCESS conference on Computers and accessibility, 203-210.

20. Harriet McBryde Johnson. 2003. The disability gulag. The New York Times Magazine 23.

21. Alison Kafer. 2013. Feminist, queer, crip. Indiana University Press.

22. Christine Kelly. 2013. Building bridges with accessible care: Disability studies, feminist care scholarship, and beyond. Hypatia 28, 4: 784-800.

23. Naomi Kimbell. 2018. Institutionalization, eugenics, and the legacy of the Boulder River School. Missoula Independent. Retrieved from https://missoulanews.com/coverstory/institutionalizati on-eugenics-and-the-legacy-of-the-boulder-riverschool/article_e66b99f6-69c4-11e8-8c99b773e9337513.html

24. Masatomo Kobayashi, Shoma Arita, Toshinari Itoko, Shin Saito, and Hironobu Takagi. 2015. Motivating multi-generational crowd workers in social-purpose work. In Proceedings of the 18th ACM Conference on Computer Supported Cooperative Work \& Social Computing, 1813-1824.

25. Nomy Lamm. This Is Disability Justice. The Body is Not An Apol. Retrieved April 12, 2018 from https://thebodyisnotanapology.com/magazine/this-isdisability-justice/ 
26. Hanlin Li, Disha Bora, Sagar Salvi, and Erin Brady. 2018. Slacktivists or Activists?: Identity Work in the Virtual Disability March. In Proceedings of the 2018 CHI Conference on Human Factors in Computing Systems, 225.

27. Peng Liu, Xianghua Ding, and Ning Gu. 2016. "Helping Others Makes Me Happy": Social Interaction and Integration of People with Disabilities. In Proceedings of the 19th ACM Conference on Computer-Supported Cooperative Work \& Social Computing (CSCW '16), 1596-1608. https://doi.org/10.1145/2818048.2819998

28. Paul K. Longmore and Lauri Umansky. 2001. The new disability history: American perspectives. NYU Press.

29. Jack M. Loomis, Reginald G. Golledge, Roberta L. Klatzky, Jon M. Speigle, and Jerome Tietz. 1994. Personal guidance system for the visually impaired. In Proceedings of the first annual ACM conference on Assistive technologies, 85-91.

30. Jennifer Mankoff, Gillian R. Hayes, and Devva Kasnitz. 2010. Disability studies as a source of critical inquiry for the field of assistive technology. In Proceedings of the 12th international ACM SIGACCESS conference on Computers and accessibility, 3-10.

31. Lucas Matney. 2018. Aira's new smart glasses give blind users a guide through the visual world | TechCrunch. Retrieved from https://techcrunch.com/2018/03/27/airas-new-smartglasses-give-blind-users-a-guide-through-the-visualworld/

32. Laura Mauldin. 2017. A Feminist Technoscientific Approach to Disability and Caregiving in the Family. In Disabling Domesticity. Springer, 139-161.

33. Ellen McCarthy and Kayla Epstein. 2018. Did they make a difference? One year later, Women's March attendees look back. Washington Post. Retrieved from https://www.washingtonpost.com/lifestyle/style/fromthe-march-to-metoo-women-lookback/2018/01/18/c5a5680a-f8bd-11e7-a9e3ab18ce41436a_story.html

34. Janis Lena Meissner, John Vines, Janice McLaughlin, Thomas Nappey, Jekaterina Maksimova, and Peter Wright. 2017. Do-It-Yourself Empowerment as Experienced by Novice Makers with Disabilities. In Proceedings of the 2017 Conference on Designing Interactive Systems, 1053-1065.

35. Mia Mingus. 2010. Interdependency (exerpts from several talks). Leaving Evidence. Retrieved March 27, 2018 from https:/leavingevidence.wordpress.com/2010/01/22/int erdependency-exerpts-from-several-talks/

36. Mia Mingus. 2010. Reflections On An Opening: Disability Justice and Creating Collective Access in Detroit. Leaving Evidence. Retrieved March 27, 2018 from

https://leavingevidence.wordpress.com/2010/08/23/ref lections-on-an-opening-disability-justice-and-creatingcollective-access-in-detroit/
37. Mia Mingus. Access Intimacy, Interdependence and Disability Justice. Leaving Evidence. Retrieved March 27, 2018 from https://leavingevidence.wordpress.com/2017/04/12/acc ess-intimacy-interdependence-and-disability-justice/

38. Ingunn Moser. 2000. Against normalisation: subverting norms of ability and disability. Science as culture 9, 2: 201-240.

39. Ingunn Moser. 2006. Disability and the promises of technology: Technology, subjectivity and embodiment within an order of the normal. Information, Communication \& Society 9, 3: 373-395.

40. Ingunn Moser. 2011. Dementia and the limits to life: anthropological sensibilities, STS interferences, and possibilities for action in care. Science, Technology, \& Human Values 36, 5: 704-722.

41. Ingunn Moser and John Law. 1999. Good passages, bad passages. The Sociological Review 47, S1: 196-219.

42. Michael Oliver. 1990. The individual and social models of disability. Oliver.

43. Halley Profita, Reem Albaghli, Leah Findlater, Paul Jaeger, and Shaun K. Kane. 2016. The AT effect: how disability affects the perceived social acceptability of head-mounted display use. In Proceedings of the 2016 CHI Conference on Human Factors in Computing Systems, 4884-4895.

44. Halley P. Profita, Abigale Stangl, Laura Matuszewska, Sigrunn Sky, and Shaun K. Kane. 2016. Nothing to hide: aesthetic customization of hearing aids and cochlear implants in an online community. In Proceedings of the 18th International ACM SIGACCESS Conference on Computers and Accessibility, 219-227.

45. Daniela K. Rosner, Hidekazu Saegusa, Jeremy Friedland, and Allison Chambliss. 2015. Walking by drawing. In Proceedings of the 33rd Annual ACM conference on human factors in computing systems, 397-406.

46. Richard K. Scotch. 2000. Disability policy: An eclectic overview. Journal of Disability Policy Studies 11, 1: 611.

47. Joseph P. Shapiro. 1994. No pity: People with disabilities forging a new civil rights movement. Three Rivers Press.

48. Kristen Shinohara and Jacob O. Wobbrock. 2011. In the shadow of misperception: assistive technology use and social interactions. In Proceedings of the SIGCHI Conference on Human Factors in Computing Systems, 705-714.

49. Kristen Shinohara and Jacob O. Wobbrock. 2016. Selfconscious or self-confident? A diary study conceptualizing the social accessibility of assistive technology. ACM Transactions on Accessible Computing (TACCESS) 8, 2: 5.

50. Sins Invalid. 2017. Skin, Tooth, and Bone-The Basis of Movement is Our People: A Disability Justice Primer. Reproductive health matters 25, 50: 149. 
51. Kiley Sobel, Kyle Rector, Susan Evans, and Julie A. Kientz. 2016. Incloodle: evaluating an interactive application for young children with mixed abilities. In Proceedings of the 2016 CHI Conference on Human Factors in Computing Systems, 165-176.

52. Susan Leigh Star and Anselm Strauss. 1999. Layers of silence, arenas of voice: The ecology of visible and invisible work. Computer supported cooperative work (CSCW) 8, 1-2: 9-30.

53. Saiganesh Swaminathan, Kotaro Hara, and Jeffrey $P$. Bigham. 2017. The Crowd Work Accessibility Problem. In Proceedings of the 14th Web for All Conference on The Future of Accessible Work, 6.

54. Anja Thieme, Cynthia L. Bennett, Cecily Morrison, Edward Cutrell, and Alex S. Taylor. 2018. "I can do everything but see!" - How People with Vision Impairments Negotiate their Abilities in Social Contexts. In Proceedings of the 36th Annual ACM Conference on human factors in computing systems (CHI'18).

55. Aditya Vashistha, Erin Brady, William Thies, and Edward Cutrell. 2014. Educational content creation and sharing by low-income visually impaired people in India. In Proceedings of the Fifth ACM Symposium on Computing for Development, 63-72.

56. Aditya Vashistha, Pooja Sethi, and Richard Anderson. 2018. BSpeak: An Accessible Crowdsourcing Marketplace for Low-Income Blind People.

57. Susan Wendell. 1997. Toward a feminist theory of disability. The disability studies reader 260: 243-256.

58. Glen W. White, Jamie Lloyd Simpson, Chiaki Gonda, Craig Ravesloot, and Zach Coble. 2010. Moving from independence to interdependence: A conceptual model for better understanding community participation of centers for independent living consumers. Journal of Disability Policy Studies 20, 4: 233-240.

59. Michele A. Williams, Caroline Galbraith, Shaun K. Kane, and Amy Hurst. 2014. Just let the cane hit it: how the blind and sighted see navigation differently. In Proceedings of the 16th international ACM SIGACCESS conference on Computers \& accessibility, 217-224.

60. Jacob O. Wobbrock, Shaun K. Kane, Krzysztof Z. Gajos, Susumu Harada, and Jon Froehlich. 2011. Ability-based design: Concept, principles and examples. ACM Transactions on Accessible Computing (TACCESS) 3, 3: 9.

61. Limin Zeng and Gerhard Weber. 2015. A pilot study of collaborative accessibility: How blind people find an entrance. In Proceedings of the 17th International Conference on Human-Computer Interaction with Mobile Devices and Services, 347-356.

62. Yu Zhong, Walter S Lasecki, Erin Brady, and Jeffrey $\mathbf{P}$ Bigham. 2015. Regionspeak: Quick comprehensive spatial descriptions of complex images for blind users. In Proceedings of the 33rd Annual ACM Conference on Human Factors in Computing Systems, 2353-2362.
63. Yu Zhong, Walter S Lasecki, Erin Brady, and Jeffrey $\mathrm{P}$ Bigham. 2015. Regionspeak: Quick comprehensive spatial descriptions of complex images for blind users. In Proceedings of the 33rd Annual ACM Conference on Human Factors in Computing Systems, 2353-2362.

64. Annuska Zolyomi, Anushree Shukla, and Jaime Snyder. 2017. Technology-Mediated Sight: A Case Study of Early Adopters of a Low Vision Assistive Technology. In Proceedings of the 19th International ACM SIGACCESS Conference on Computers and Accessibility, 220-229.

65. Kathryn Zyskowski, Meredith Ringel Morris, Jeffrey P. Bigham, Mary L. Gray, and Shaun K. Kane. 2015. Accessible crowdwork?: Understanding the value in and challenge of microtask employment for people with disabilities. In Proceedings of the 18th ACM Conference on Computer Supported Cooperative Work \& Social Computing, 1682-1693.

66. 2017. Women's March. Retrieved from http://www.womensmarch.com

67. Define Independent at Dictionary.com. Dictonary.com. Retrieved March 19, 2018 from http://www.dictionary.com/browse/independent

68. BeMyEyes. Retrieved from bemyeyes.org 